\title{
PREVENÇÃO AO USO ABUSIVO DE ÁLCOOL NO CONTEXTO ESCOLAR: RELATO DE EXPERIÊNCIA DO PROJETO "RECRIANDO CAMINHOS"
}

\author{
Marcelle Aparecida de Barros Junqueira ${ }^{1}$ \\ Maria José Nunes ${ }^{2}$ \\ Frank José Silveira Miranda ${ }^{3}$ \\ Vanessa Gonçalves Castro ${ }^{4}$ \\ Danielle Campos Massa ${ }^{4}$ \\ Ana Cristina Gennari Bernardes ${ }^{4}$ \\ Rosana de Oliveira Santos ${ }^{4}$ \\ Neirielen Francisco da Silva ${ }^{4}$ \\ Ludmila Alves Ferreira ${ }^{4}$
}

\begin{abstract}
RESUMO: Atualmente, o uso de álcool apresenta enormes impactos sociais, físicos e mentais, a ponto de ser considerado, pela Organização Mundial de Saúde (OMS), um problema de saúde pública. Estudos epidemiológicos apontam que o consumo de álcool vem acontecendo em idade cada vez mais precoce, inclusive no Brasil. Assim, este relato visa descrever ações de um projeto que visou desenvolver ações preventivas ao uso abusivo de álcool por estudantes de uma escola pública no município de Uberlândia/MG. As estratégias de atuação foram oficinas educativas, detecção do consumo de álcool problemático, intervenção em casos detectados como uso nocivo e encaminhamento de casos mais graves. Observou-se que a escola é um terreno fértil para ações de prevenção ao uso abusivo de álcool.
\end{abstract}

PALAVRAS-CHAVE: Educação em saúde. Saúde Escolar. Consumo de bebidas alcoólicas.

Alcohol abuse prevention in the school context: reporting of project experience "Recreating Paths"

\begin{abstract}
Currently the use of alcohol has enormous social impacts, physical and mental to the point of being considered a public health problem by the World Health Organization. Epidemiological studies indicate that alcohol consumption has been happening increasingly early age including in Brazil. Thus, this living report aimed the project to develop preventive actions to alcohol abuse among students from a public school in Uberlândia city, MG. The operational strategies was educational workshops, detection of problematic alcohol consumption, intervention in cases detected as harmful use and forwarding of the most serious cases. It's been realized that the school is a breeding ground for preventive actions to alcohol abuse.
\end{abstract}

KEYWORDS: Health education. School health. Consumption of alcoholic beverages.

\footnotetext{
${ }^{1}$ Doutora em Enfermagem Psiquiátrica pela Universidade de São Paulo, professora adjunta no curso de Enfermagem da Faculdade de Medicina da Universidade Federal de Uberlândia (marcellebarros@famed.ufu.br).

${ }^{2}$ Mestre em Enfermagem pela Universidade Federal de Minas Gerais, professora assistente no curso de Enfermagem da Faculdade de Medicina da Universidade Federal de Uberlândia (mjnunes2000@yahoo.com.br).

3 Doutorando em Enfermagem pela Universidade Estadual de Campinas, professor no curso de Enfermagem da Faculdade de Medicina da Universidade Federal de Uberlândia (frankenfermeiro@yahoo.com.br).

${ }^{4}$ Acadêmicas do curso de Enfermagem da Faculdade de Medicina da Universidade Federal de Uberlândia (vanessagcastro@enf.ufu.br; danielle massa@hotmail.com; tinabernardes@yahoo.com.br; rosana_o_s@hotmail.com; neyriellen@yahoo.com.br; ludmilalvesferreira@hotmail.com). 


\section{INTRODUÇÃO}

Segundo a Organização Mundial de Saúde, considerando pessoas de todas as idades e todos os locais do mundo, o uso de álcool está associado a 1,3\% da carga geral de anos de vida perdidos por incapacidade ou morte relacionada ao uso abusivo de drogas. Para homens, a taxa quase dobra. Se considerarmos apenas a incapacidade para pessoas entre 15 e 44 anos de idade, de ambos os sexos, o álcool é responsável por 5,5\% de perda de anos por incapacidade, sendo esta a segunda causa de perda mais importante (OMS, 2001).

O relatório da OMS mostra, também, que os gastos hospitalares com os problemas de saúde provocados pelo álcool ultrapassam a arrecadação com impostos sobre o álcool (OMS, 2001). Os danos pelo uso dessa substância são claramente relacionados ao padrão de consumo, sendo eles a quantidade e a frequência. Assim, quanto mais uma pessoa bebe, maior o risco de provocar ou sofrer danos. Isto se observa, por exemplo, nos estudos internacionais que comprovam a relação entre os acidentes de trânsito e o consumo da bebida alcoólica (BABOR et al., 2003).

Atualmente, os transtornos relacionados ao uso de álcool e outras drogas não classificam mais os indivíduos como dependentes e não dependentes. Dessa forma, qualquer padrão de consumo pode trazer problemas ao indivíduo. Assim, classifica-se como consumo de baixo risco aquele que apresenta baixas doses, cercado das precauções necessárias à prevenção de acidentes relacionados (LARANJEIRA et al., 2003).

Já o uso nocivo de álcool é utilizado para indivíduos que bebem eventualmente, mas são incapazes de controlar ou adequar seu modo de consumo. Isto pode levar aos problemas sociais (brigas, falta de emprego), físicos (acidentes) e psicológicos (heteroagressividade). Finalmente, a dependência ao álcool é caracterizada pelo consumo compulsivo e destinado a evitar os sintomas de abstinência e cuja intensidade é capaz de ocasionar problemas sociais, físicos e ou psicológicos (LARANJEIRA et al., 2003).

Dados do Levantamento Nacional sobre os padrões do consumo de álcool na população brasileira demonstram que 52\% dos brasileiros adultos maiores de 18 anos bebem (pelo menos 1 vez por ano); e que $12 \%$ da população brasileira têm um uso nocivo e dependente de álcool; o que representa um bom índice em termos de saúde pública para que se possa dimensionar o custo social do álcool (BRASIL, 2007).

Nesse Levantamento, encontrou-se uma alta frequência de adolescentes (9\%) que bebem mais do que uma vez por semana (12\% meninos e $6 \%$ meninas). Em relação à dose usual, quase $50 \%$ dos meninos bebeu mais do que três doses por situação habitual e cerca de um terço deles consumiu cinco doses ou mais. Em relação ao binge drinking (beber episódico, durante o qual uma grande soma de bebidas é consumida em um curto período de tempo), os adolescentes apresentaram altas taxas, com $21 \%$ dos meninos e $12 \%$ das meninas (BRASIL, 2007).

Quando se compara jovens entre 18 e 25 anos com adolescentes, as diferenças são significativas quanto à idade de experimentação e do uso regular. No início do consumo, os adolescentes 
começaram aos 13,9 anos de idade e os adultos jovens aos 15,3 anos. O uso regular pelos adolescentes começou aos 14,8 anos e pelos adultos jovens aos 17,3 anos. Esses números foram obtidos depois de desconsiderar jovens adultos que haviam iniciado o consumo após os 18 anos, ou seja, já controlando para uma redução nas idades médias dos jovens adultos (BRASIL, 2007).

O consumo de bebidas alcoólicas por adolescentes traz consequências negativas diversas, tais como: problemas no estudo, desajustes sociais, prática de sexo sem proteção e/ou sem consentimento, risco maior de suicídio ou homicídio e acidentes relacionados ao consumo de álcool (FADEN, 2005 apud LARANJEIRA, 2003). Pesquisas apontam que começar a beber em idade precoce é um fator que influenciará problemas futuros com o álcool (MAGGS; SCHULENBEG, 2005 apud LARANJEIRA, 2003).

A prevenção de drogas visa à adoção de uma atitude responsável com relação aos psicotrópicos. O objetivo último da precaução, no campo dos problemas relacionados ao consumo de drogas psicotrópicas, é procurar que os membros de uma dada população não abusem de drogas e, consequentemente, não causem danos pessoais e sociais relacionados ao uso excessivo, nem prejuízos que daí possa decorrer (CARLINI; CARLINI-COTRIM; SILVA FILHO, 1990). Assim, os tipos de prevenção dividem-se em:

Prevenção primária: constituída de ações antecipatórias que visam diminuir a probabilidade do início ou do desenvolvimento de uma condição. No domínio das substâncias psicoativas, o objetivo é impedir que se produza um consumo problemático antes mesmo do primeiro uso. Incluem-se, aqui, a educação para a promoção da saúde, a informação e medidas sociais e legais.

Prevenção secundária: consiste em intervenções para se evitar que um estado de dependência se estabeleça. Este tipo de trabalho é definido como uma intervenção especializada endereçada a pessoas que já manifestaram sinais de certa dificuldade com as substâncias psicoativas, em razão de um uso impróprio ou nocivo, dirigindo-se, portanto, a uma população bem definida, ou seja, àquela que dá sinais de excessos esporádicos ou abuso momentâneo. Aqui se incluem o diagnóstico precoce e as estratégias de Intervenção Breve (IBs).

Prevenção terciária: consiste em quaisquer atos destinados a diminuir a prevalência das incapacidades crônicas em uma população, reduzindo-se ao mínimo as deficiências funcionais consecutivas à doença, no caso, a dependência de substâncias psicoativas. Aqui se incluem medidas terapêuticas propriamente ditas e reabilitação.

A escola é um campo social permeável às abordagens transdisciplinares e à profusão de abordagens de diferentes correntes teóricas em todas as áreas de conhecimento. Portanto, a escola é um território privilegiado para a incorporação de conhecimentos sobre saúde, fomento a atitudes e práticas de vida saudáveis, assim como para a possibilidade de transformar o atual quadro de vulnerabilidade social que muitos jovens brasileiros vivem atualmente, incluindo o uso abusivo de álcool e outras drogas (PAIVA; PUPOL; BARBOZA, 2006).

Considerando a necessidade de uma abordagem concreta no tocante a prevenção ao uso excessivo de álcool, principalmente na população jovem, o projeto "Recriando Caminhos" buscou se 
aproximar da realidade direta desses cidadãos por meio do contexto escolar, visando, antes de tudo, contribuir para que possam reafirmar condutas de vida saudáveis. Além do mais, no caso do jovem que já apresentava indicativos de estar ultrapassando a tênue linha entre o beber "socialmente" e o beber "problemático", o projeto pode colaborar para que ele vá RECRIANDO CAMINHOS na direção de atitudes mais seguras e responsáveis.

\section{OBJETIVOS}

Os objetivos do projeto foram:

a. Fomentar ações de caráter preventivo, principalmente nos níveis primário e secundário, acerca do uso excessivo de bebida alcoólica.

b. Induzir a redução dos níveis de consumo de bebidas alcoólicas entre estudantes do oitavo ano do Ensino Fundamental ao terceiro ano do Ensino Médio de uma escola pública.

c. Ampliar o debate permanente dentro do ambiente escolar (alunos, pais, professores e demais integrantes) sobre o consumo de bebida alcoólica e suas consequências.

d. Ativar o surgimento de multiplicadores de ações preventivas ao uso abusivo de álcool na comunidade escolar e entre os parceiros dos órgãos participantes.

e. Expandir os conhecimentos crítico e reflexivo sobre o hábito de consumo do álcool e suas possíveis consequências entre os estudantes.

f. Prevenir, a médio e longo prazo, o surgimento de novos casos de dependência ao álcool.

\section{METODOLOGIA}

O projeto "Recriando Caminhos" desenvolveu suas atividades em três momentos:

Oficinas educativas: destinadas aos estudantes, procuraram seguir os preceitos da chamada pedagogia da problematização. Essa aderência deve-se ao fato de que pensar em promoção da saúde é falar sobre incremento do poder (empowerment) comunitário e pessoal, por meio de desenvolvimento de habilidades e atitudes, conducentes à aquisição de poder técnico (saberes) e político para atuar em prol de sua saúde, como propõe a Carta de Ottawa (PEREIRA, 2003).

Assim, as estratégicas metodológicas das oficinas foram: grupos de discussão, dramatizações, debates, discussão de casos, uso de áudio visual (filmes, data show, slides, TV, DVD), música, vivências, dinâmicas de grupo e outros. 
Os temas das oficinas abordaram assuntos como aspectos sociais e culturais do hábito de beber no âmbito da sociedade atual, a relação do jovem com o consumo de álcool, os limites do beber "socialmente", as consequências sociais do uso excessivo de bebida alcoólica, as consequências físicas e emocionais do uso excessivo de bebida alcoólica, o alcoolismo, entre outros assuntos.

Detecção dos níveis de consumo de bebidas alcoólicas entre os estudantes: foi utilizado o teste de Identificação do Uso do Álcool (AUDIT), preconizado pela Organização Mundial de Saúde para esse fim: trata-se de um instrumento de fácil aplicação e validação transcultural utilizado em diferentes serviços (BARBOR et al., 2001).

Intervenção junto a estudantes com sinais indicativos de uso abusivo de álcool: foi realizado, por meio da estratégia das Intervenções Breves preconizadas pela OMS. As IBs objetivam, primariamente, detectar o problema e motivar o paciente a alcançar determinadas ações, como por exemplo, iniciar um tratamento ou, ainda, melhorar seu nível de informação sobre riscos associados ao uso de substâncias, por meio de um aumento de seu senso de risco e de autocuidado (MARQUES; FURTADO, 2004).

As IBs não exigem muito tempo e se incorporam, com facilidade, à consulta usual de médicos de família, enfermeiros e agentes de saúde comunitária. Podem durar de cinco a trinta minutos, pelo menos, e são constituídas por uma curta sequência de etapas que incluem a identificação e dimensionamento dos problemas ou dos riscos - geralmente por meio do uso de um instrumento padronizado de rastreamento, como o AUDIT, por exemplo - e o oferecimento de aconselhamento, orientação e, em algumas situações, monitoramento periódico do sucesso em atingir metas assumidas voluntariamente pelo paciente (MARQUES; FURTADO, 2004).

\section{RESULTADOS E DISCUSSÃO}

No mês de março de 2010 foi realizado, no campus Umuarama da Universidade Federal de Uberlândia, um curso teórico-prático sobre o uso abusivo de álcool e intervenções básicas, com carga horária de 36 horas, oferecido a todos os graduandos do curso de Enfermagem da Faculdade de Medicina. Logo após o término deste, ocorreu um processo seletivo para a seleção de seis alunos para participarem do projeto "Recriando Caminhos".

O projeto ocorreu no período de maio a novembro de 2010. Inicialmente, aconteceram reuniões com a direçãodeuma escolapública estadualde Uberlândia-MG, afimdeelaborarum cronogramaa serseguido.

Oprojeto aconteceu quatro vezes porsemana, no período matutino, simultaneamente com três turmas do $8^{\circ}$ ano ano, três turmas do $9^{\circ}$ ano do Ensino Fundamental; duas turmas do $1^{\circ}$, duas do $2^{\circ}$ e uma do $3^{\circ}$ ano doEnsino Médioe uma turma doEnsino de Jovense Adultos. Aotodo, oprojeto abrangeu 420 estudantes.

Inicialmente, foram utilizadas dinâmicas como estratégias de ensino-aprendizagem, criando um ambiente propício para a interação entre integrantes do projeto e estudantes da escola, permitindo 
aos alunos uma maior reflexão sobre hábitos saudáveis de vida e os problemas causados pela utilização da bebida alcoólica.

Ocorreram oficinas em todas as salas de aula sobre os tópicos: O que é saúde? O que é doença? O que fazer para se ter saúde? A sala foi dividida em grupos de aproximadamente cinco alunos. Distribuímos tesouras sem ponta, colas, revistas, cartolinas e pincéis anatômicos para que discorrerem seus conhecimentos sobre estes temas.

Em um segundo momento, utilizamos como critério de avaliação sobre o consumo de bebida alcoólica o AUDIT. O teste foi realizado na forma de entrevista individual entre os alunos do $7^{\circ}$ ano do Ensino Fundamental ao $3^{\circ}$ ano do Ensino Médio. O teste foi aplicado na sala dos professores, a fim de que não houvesse divulgação dos resultados entre os alunos, ou seja, cada aluno soube seu escore isoladamente.

No terceiro momento, foi realizado um amplo debate sobre o álcool e suas intercorrências em todas as salas de aula. Os alunos demonstraram bastante interesse e foram colaborativos. Foi um momento ímpar, de reflexão interna, que favoreceu o engajamento dos alunos e observação de diferentes perspectivas do mundo atual.

No quarto momento, foi realizada a intervenção em alunos identificados com o uso nocivo de bebidas alcoólicas por meio do AUDIT. Foi enviada uma carta destinada para cada estudante, com o dia, o horário e o local para a realização da intervenção. Foram explicadas as consequências do ato abusivo de beber e fornecidas informações de como diminuir o uso e/ou abster-se, com a adoção de algumas condutas simples, tendo como o objetivo a redução do consumo de bebida alcoólica, como por exemplo: beber mais lentamente, comer antes de beber, alternar bebidas alcoólicas e bebidas sem álcool.

O projeto apresentou algumas limitações e não conseguiu atingir o objetivo de envolver os pais na discussão sobre a temática do uso de álcool, ocorrida pela não adesão dos mesmos a uma reunião solicitada pela equipe executora do projeto. Uma das hipóteses que pode explicar o fato ocorrido foi a mudança na direção da escola, acontecida exatamente no mesmo período do projeto, o que dificultou o entendimento do projeto pela nova direção e, consequente, ajuda no engajamento dos pais e outros servidores da escola.

Nesse mesmo sentido, o projeto também se propôs a formar multiplicadores dessas informações entre os próprios estudantes que participaram das oficinas e que pudessem repassar essas informações a outros jovens. Contudo, houve algumas dificuldades relacionadas aos horários de aula para desenvolver as atividades, fazendo com que o cronograma fosse alterado. Devido a isso, não restou tempo suficiente para reforçar essa ideia com os alunos.

A mudança no cronograma de atividades também impediu que ações do projeto extrapolassem o âmbito escolar e abrangesse outros cenários, tais como serviços de saúde, segurança, assistência social, áreas de lazer, entre outros.

A escola é um celeiro de muitas possibilidades e isto se observa também em relação às várias 
maneiras de prevenir o uso de álcool e outras drogas dentro desse ambiente. Nesta perspectiva, devemos atentar para uma mudança significativa no enfoque, substituindo a abordagem repressora pela de orientação aos jovens, além de criar espaços para discussões. Quanto mais realizados e conscientes os jovens estiverem, menores serão os riscos de terem problemas com álcool e outras drogas (SCIVOLETTO; FERREIRA, 2002).

Mesmo que não tenha ocorrido um trabalho diretamente com a família, devido às limitações descritas, acredita-se que isso seja de suma importância, pois a conversa com os pais é uma ação importante. Escola e família, juntas, encontram meios para ajudar a criança e o adolescente. Trata-se, aqui, de analisar a questão de forma ampla, considerando os aspectos envolvidos no uso de drogas: a pessoa, a droga e o contexto. A escola e a família são partes essenciais deste contexto (SCIVOLETTO; FERREIRA, 2002).

Considerando, ainda, que o uso do álcool e de outras drogas está associado a diversos outros fatores de risco, acarretando prejuízos à saúde e à vida dos adolescentes, torna-se urgente a ação das famílias, escolas e sociedade para traçar medidas de promoção à saúde e prevenção do uso destas substâncias (MALTA et al.,2011).

Na educação para a saúde, é importante ajudar/ensinar os adolescentes a desenvolverem competências que lhes permitam resistir à influência dos amigos para o envolvimento em comportamentos que coloquem em risco a sua saúde e bem-estar. Essas ações devem se desenvolver na escola e na comunidade, e o envolvimento dos pares e dos pais é importante para o seu sucesso, dado o papel que eles têm na modelação de comportamentos (FERREIRA; TORGAL, 2010).

A escola apresenta-se como um excelente espaço de atividades de prevenção ao consumo de álcool em seus diferentes níveis. Além de os alunos passarem grande parte do seu tempo neste espaço, ainda reforçam-se valores e normas sociais. Educação e saúde auxiliam na construção de indivíduos e coletividades que constituem a sociedade (LOPES et al.,2007).

O processo educacional, além de gerar e disseminar conhecimentos, ainda possibilita uma ampliação do saber na dimensão humana e de melhoria da qualidade de vida. Nesse ambiente escolar, deve-se também "aprender a ser" e "aprender a conviver". Com isso, entendemos que a promoção da saúde é uma ação com grande potencial para desenvolvimento em espaços escolares, já que estes são locais de diálogos, privilegiados para troca de saberes e expressão da diversidade cultural (BRASIL, 2005).

\section{CONSIDERAÇÕES FINAIS}

O desenvolvimento do projeto foi uma experiência muito valiosa para os graduandos em Enfermagem, os quais puderam perceber o despertar de interesse por parte dos estudantes da escola pública sobre os problemas acarretados ao uso do álcool. Com eles, os futuros enfermeiros tiveram uma intensa troca de experiências e conhecimentos com toda a comunidade escolar e também adquiriram conhecimentos durante a preparação e realização das atividades. 
Alguns objetivos importantes do projeto não puderam ser alcançados, mas merecem ser retomados devido ao fato de serem extremamente pertinentes, como: envolvimento da família de demais setores da comunidade e a formação e acompanhamento específico de multiplicadores.

Dessa forma, conclui-sequeoprojetodevesercontínuonessaescolae se expandirpara outrasinstituições, visto que há muitos adolescentes que utilizam bebida alcoólica de forma exagerada, e também por levar a uma reflexão do consumo de álcool pelos próprios universitários que executam as atividades.

Aescola merece maior enfoque dos profissionais da saúde, uma vez que ela proporciona conhecimento, educação e favorece o crescimento intelectual e pessoal. O enfermeiro, que dentre as suas competências e habilidades estão as que envolvem a educação, deveria abranger também a área da educação básica, uma vez que ele proporcionaria os pilares para o indivíduo crescer e se desenvolver com saúde.

\section{REFERENCIAS}

BARBOR T. et al. AUDIT - the alcohol use disorders identification test: guidelines for use in primary care. Geneva: World Health Organization; 2001.

BRASIL. Ministério da Saúde. Censo Escolar 2005: levantamento das ações em DST/AIDS: saúde sexual e reprodutiva e drogas. Disponível em: <http://www.aids.gov.br $>$. Acesso em: 10 mar. 2012.

Levantamento Nacional do sobre os padrões do consumo de álcool na população brasileira. Brasília: Secretaria Nacional Antidrogas, 2007.

CARLINI, E. A.; CARLINI-COTRIM, B. H. R. S.; SILVA FILHO, A. R. Sugestões para programas de prevenção ao abuso de drogas no Brasil. São Paulo: EMP/CEBRID, 1990.

FERREIRA, M. M. S. R. S; TORGAL, M. C. L. F. P. R. Consumo de tabaco e de álcool na adolescência. Rev. Latino-Am. Enfermagem, Ribeirão Preto, v. 18, n. 2, p. 255-261, mar.-abr. 2010 .

LARANJEIRA, R. et al. Usuários de substâncias psicoativas: abordagem, diagnóstico e tratamento. 2 ed. São Paulo: Conselho Regional de Medicina de São Paulo/ Associação Médica Brasileira, 2009.

LOPES, G. T. et al.O enfermeiro no ensino fundamental: desafios na prevenção ao consumo de álcool. Esc. Anna Nery Rev. Enferm., Rio de Janeiro, v.11, n.4, p.712 -716, dez. 2007,

MALTA, D. C. et al. Prevalência do consumo de álcool e drogas entre adolescentes: análise dos dados da Pesquisa Nacional de Saúde Escolar. Rev Bras Epidemiol., São Paulo, v.1, n.1, p.136-146, 2011.

MARQUES, A. C. P. R.; FURTADO, E. F. Intervenções breves para problemas relacionados ao álcool. Rev. Bras. Psiquiatr., São Paulo, v. 26, p. 28-32, 2004. 
OMS. Intervensión breve para el consumo de riesgo y perjudicial de alcohol: um manual para la utilización en atención primaria. OMS: Departamento de Salud Mental y Dependência de Substancias, 2001.

PAIVA, V; PUPOL; L.R, BARBOZA; R. O direito à prevenção e os desafios da redução da vulnerabilidade ao HIV/AIDS no Brasil. Rev. Saúde Pública, São Paulo, v. 40, 109-119, abr. 2006.

PEREIRA. A. F. As tendências pedagógicas e a prática educativa nas ciências da saúde. Cad. Saúde Pública, Rio de Janeiro, v. 19, n. 5, p. 1527-1534, 2003.

SCIVOLETTO, S.; FERREIRA R. C. A prevenção ao uso indevido de drogas na escola. In: Formação de multiplicadores de informações preventivas sobre drogas. Brasília (DF): SENAD, 2002. 